\title{
Planetesimal formation by an axisymmetric radial bump of the column density of the gas in a protoplanetary disk
}

Isamu K. Onishi ${ }^{1}$ and Minoru Sekiya ${ }^{2^{*}}$

\begin{abstract}
We investigate the effect of a radial pressure bump in a protoplanetary disk on planetesimal formation. We performed the two-dimensional numerical simulation of the dynamical interaction of solid particles and gas with an initially defined pressure bump under the assumption of axisymmetry. The aim of this work is to elucidate the effects of the stellar vertical gravity that were omitted in a previous study. Our results are very different from the previous study, which omitted the vertical gravity. Because dust particles settle toward the midplane because of the vertical gravity to form a thin dust layer, the regions outside of the dust layer are scarcely affected by the back-reaction of the dust. Hence, the gas column density keeps its initial profile with a bump, and dust particles migrate toward the bump. In addition, the turbulence due to the Kelvin-Helmholtz instability caused by the difference of the azimuthal velocities between the inside and outside of the dust layer is suppressed where the radial pressure gradient is reduced by the pressure bump. The dust settling proceeds further where the turbulence is weak, and a number of dust clumps are formed. The dust density in some clumps exceeds the Roche density. Planetesimals are considered to be formed from these clumps owing to the self-gravity.
\end{abstract}

Keywords: Planetary systems, Protoplanetary disks, Solar system formation, Hydrodynamics, Instabilities, Planetesimal, Formation of planets, Streaming instability, Gravitational instability, Dust

\section{Introduction}

A hypothetical small solid body with a radius $\gtrsim 1 \mathrm{~km}$ formed in a protoplanetary disk is called a planetesimal. Planetesimals grow by collisional coagulation owing to mutual gravity (Wetherill and Stewart 1989; Kokubo and Ida 1996, 1998). The formation processes of planetesimals from dust particles are not fully understood (Chiang and Youdin 2010). There are two major theories for the formation of planetesimals. One is the mutual sticking of dust aggregates (Weidenschilling and Cuzzi 1993), and another is the gravitational instability of the dust layer (Safronov 1969; Goldreich and Ward 1973). In this work, we restrict ourselves to considering the formation of planetesimals through the gravitational instability in a

\footnotetext{
*Correspondence: sekiya.minoru.393@m.kyushu-u.ac.jp

2 Department of Earth and Planetary Sciences, Faculty of Science, Kyushu

University, 744, Motooka, Nishi-ku, Fukuoka 819-0395, Japan

Full list of author information is available at the end of the article
}

dead zone where the ionization fraction is not sufficient for the magneto-rotational instability to drive turbulence.

Even in a dead zone, the velocity gradient vertical to the midplane causes Kelvin-Helmholtz instability (Weidenschilling 1980; Cuzzi et al. 1993; Sekiya 1998), and the relative motion of dust and gas attributed to the radial pressure gradient causes streaming instability (Youdin and Goodman 2005; Youdin and Johansen 2007; Johansen and Youdin 2007). The gravitational instability grows if the volume density of dust particles $\rho_{\mathrm{d}}$ (i.e., the total mass of dust particles in a unit volume of the disk) exceeds the Roche density, $\rho_{\text {Roche }}=0.85 M_{*} / r^{3}$, where $M_{*}$ is the stellar mass and $r$ is the orbital radius (Chandrasekhar 1987). The numerical simulations by Johansen et al. (2009), Bai and Stone (2010b), and Carrera et al. (2015) have shown the following results. The turbulence induced by the Kelvin-Helmholtz instability and streaming instability diffuses dust particles and prevents $\rho_{\mathrm{d}}$ from exceeding $\rho_{\text {Roche, }}$ as long as $Z \equiv \Sigma_{\mathrm{p}} / \Sigma_{\mathrm{g}} \lesssim 0.01$, where 
$\Sigma_{\mathrm{p}}$ and $\Sigma_{\mathrm{g}}$ are the column densities of the dust particles and gas, respectively (hereafter, we call $Z$ the metallicity). In contrast, particle clumps grow because of the streaming instability and $\rho_{\mathrm{d}}$ may exceed $\rho_{\text {Roche }}$ if $Z \gtrsim 0.03$ and $0.01 \lesssim \tau_{\mathrm{s}} \lesssim 1$, where the Stokes number is defined by $\tau_{s}=t_{\text {stop }} \Omega_{K}$, where $t_{\text {stop }}$ is particle's stopping time due to the gas drag force and $\Omega_{K}$ is the Keplerian angular velocity. According to Hayashi's protoplanetary disk model (Hayashi 1981), $Z=0.0042$ inside the snow line; thus, planetesimal formation by gravitational instability is unlikely.

In the usual disk model, the radial pressure gradient is negative, and gas rotates slightly more slowly than the Keplerian velocity. Dust particles that are not affected by the pressure gradient rotate faster than the gas, and particles migrate toward the star owing to the negative torque caused by the headwind (Adachi et al. 1976; Weidenschilling 1977; Nakagawa et al. 1986). If the radial pressure gradient is positive in some region of the disk, dust particles migrate outward. If there is a pressure bump in a protoplanetary disk, dust particles migrate toward the pressure maximum radius (Haghighipour and Boss 2003), and the increase of $Z$ may promote planetesimal formation. Pressure bumps may arise around the snow line where water vapor is continuously added from ice particles that cross the snow line from the outside to the inside (Kretke and Lin 2007) and the boundary of a dead zone of the magneto-rotational instability (Lyra and Mac Low 2012).

Recently, Taki et al. (2016) performed a numerical simulation of dust and gas with an initially defined pressure bump. They showed that the initially defined pressure bump evolves to become a region where the radial pressure gradient is almost zero. The radial width of the region spreads as dust particles outside the initially defined pressure bump migrate toward the region. In this region, dust particles stop migrating because the relative velocity of the dust and gas is almost zero and no exchanges of their angular momenta occur. They concluded that the streaming instability that is excited by the relative velocity of dust and gas would be difficult to grow and a pressure bump will not promote planetesimal formation. However, Taki et al. (2016) omitted two important effects: (1) non-axisymmetric vortices caused by the Rossby wave instability (Li et al. 2000; Lyra and Mac Low 2012; Meheut et al. 2012a, b; Lin 2012; Ono et al. 2016) and (2) the component of stellar gravity vertical to the midplane of the disk.

The Rossby wave instability is inevitable in a disk with a pressure bump that has an amplitude large enough for the radial pressure gradient to be positive somewhere in the bump. However, the saturated state of the instability depends on the mechanisms that cause the bump and the heating and cooling processes in the disk. Complex models that include all these effects are beyond the scope of this work.

Here, we simply assume axisymmetry and perform local two-dimensional (radial and vertical) simulations of dust and gas. This study differs from that of Taki et al. (2016) because we take the vertical component of the stellar gravity into account. We compare our results with those of Taki et al. (2016) and discuss the causes of the differences.

\section{Methods}

We use Hayashi's model (Hayashi 1981) as a base state before defining a radial bump of the gas density. We employ the local shearing box model in which $x=r-r_{0}$, where $r_{0}$ is the fiducial radius, $y$ points toward the azimuthal direction, and $z$ is vertical to the midplane $(z=0$ at the midplane). The origin moves with the velocity of the base-state gas, which is slower than the Kepler velocity $v_{K}$ by amount $\eta v_{K}$, where $\eta \equiv-\left(\partial P_{0} / \partial r\right) /\left(2 \rho_{\mathrm{g} 0} \Omega_{K}^{2} r\right)$ and $\rho_{\mathrm{g} 0}$ and $P_{0}$ are the gas density and pressure, respectively, at the midplane of the base state (Adachi et al. 1976; Weidenschilling 1977; Nakagawa et al. 1986). We omit the effect of self-gravity. Our aim is to investigate if $\rho_{d}$ exceeds $\rho_{\text {Roche }}=3.8 \times 10^{2}\left(r_{0} / 1 \mathrm{AU}\right)^{-1 / 4} \rho_{g 0}$.

We numerically solve equations of continuity and momentum of the gas, and the motion of dust particles (see equations (1)-(3) of Bai and Stone 2010b). We assume the isothermal equation of state $P=c_{s}^{2} \rho_{\mathrm{g}}$, where $\rho_{\mathrm{g}}$ is the gas density, $c_{\mathrm{s}}$ is the isothermal sound speed that is assumed constant, and $P$ is the local pressure, which does not include the global pressure gradient; the effect of the global pressure gradient is included in constant parameter $\eta$ (see Bai and Stone 2010a for details). Simulation is performed using the Athena code for particles and gas (Bai and Stone 2010a) under the axisymmetry assumption $\partial / \partial y=0$. We assume that all particles have an identical Stokes number $\tau_{\mathrm{s}}$ before the pressure bump is added. A model is then characterized by three dimensionless parameters: $\tau_{\mathrm{s}}, Z$, and $\Pi \equiv \eta v_{K} / c_{\mathrm{s}}=\eta r / H_{\mathrm{g}}$, where $H_{\mathrm{g}} \equiv c_{\mathrm{s}} / \Omega_{K}$ is the vertical scale height of the disk. We set $Z_{0}=4.2 \times 10^{-3}$ and $\Pi=0.05$ as a base state; these are representative values of Hayashi's model inside the snow line. We assume $\tau_{\mathrm{s}}=0.01$, which corresponds to $\mathrm{cm}$-sized particles in the asteroid region of Hayashi's model. We defined an initial gas density with a radial density bump identically to Taki et al. (2016) as follows:

$\rho_{\mathrm{g}}=\rho_{\mathrm{g} 0} \exp \left[-z^{2} /\left(2 H_{g}^{2}\right)\right]\left\{1+A \exp \left[-x^{2} /\left(2 W^{2}\right)\right]\right\}$,

where $A=0.2$, and $W=0.5 H_{g}=10 \eta r$. The initial dust density is $\rho_{\mathrm{d} 0}=0.1 \rho_{\mathrm{g} 0}$ in the dust layer with $|z| \leq H_{\mathrm{d} 0}$ and otherwise $\rho_{\mathrm{d} 0}=0$, where $H_{\mathrm{d} 0}=Z \Sigma_{\mathrm{g} 0} /\left(2 \rho_{\mathrm{d} 0}\right)=1.05 \eta r$ 
and $\Sigma_{\mathrm{g} 0}=(2 \pi)^{1 / 2} \rho_{\mathrm{g} 0} H_{\mathrm{g}}$. The initial azimuthal velocity of the gas is derived from radial force balances without dust and gas interaction. The simulation region is $-75 \leq x /(\eta r) \leq 75$ and $-2.5 \leq z /(\eta r) \leq 2.5$. This region is divided into $\left(N_{x}, N_{z}\right)=(24300,640)$ meshes. Superparticles that represent dust particles are distributed using uniform random numbers in the region $|z| \leq H_{\mathrm{d} 0}$; the number of particles is $N_{\mathrm{p}}=15,552,000$. The boundary conditions are shearing periodic in $x$ and reflective in $z$ (see Bai and Stone 2010b concerning the adequacy of the reflection boundary condition).

\section{Results and discussion}

In our model, the radial pressure gradient force of the base state is represented by $-\left(\rho_{\mathrm{g} 0}\right)^{-1}\left(\partial P_{0} / \partial r\right)=2 \eta v_{K} \Omega_{K}$ and the variation of the pressure gradient force from the base state is given by $-\left(\rho_{\mathrm{g}}\right)^{-1}(\partial P / \partial r)=-\left(\rho_{g}\right)^{-1}(\partial P / \partial x)$. Hence, the total pressure gradient force is the sum of these two quantities (note that $\rho_{\mathrm{g}} \approx \rho_{\mathrm{g} 0}$, because $\left.\eta r / H_{g}=\Pi \ll 1\right)$. We define the normalized radial pressure gradient force by

$$
\begin{aligned}
f & \equiv\left[2 \eta v_{K} \Omega_{K}-\left(\rho_{g}\right)^{-1}(\partial P / \partial x)\right] /\left[2 \eta v_{K} \Omega_{K}\right] \\
& =1-\left(2 \eta v_{K} \Omega_{K} \rho_{g}\right)^{-1}(\partial P / \partial x) .
\end{aligned}
$$

The distribution of $f$ at the midplane is shown in Fig. 1. Initial distributions show that $f \rightarrow 1$ in the limit of $|x| \rightarrow \infty$; in contrast, $f<0$ in the region $x_{-}<x<x_{+}$ because of the effect of the pressure bump, where $x_{-} /(\eta r)=-20.36$ and $x_{+} /(\eta r)=-3.13$ in the case of our parameter values. In this region, dust particles migrate outward and the metallicity grows with time around $x_{+}$, as shown in Fig. 2. Figure 3 shows the radial

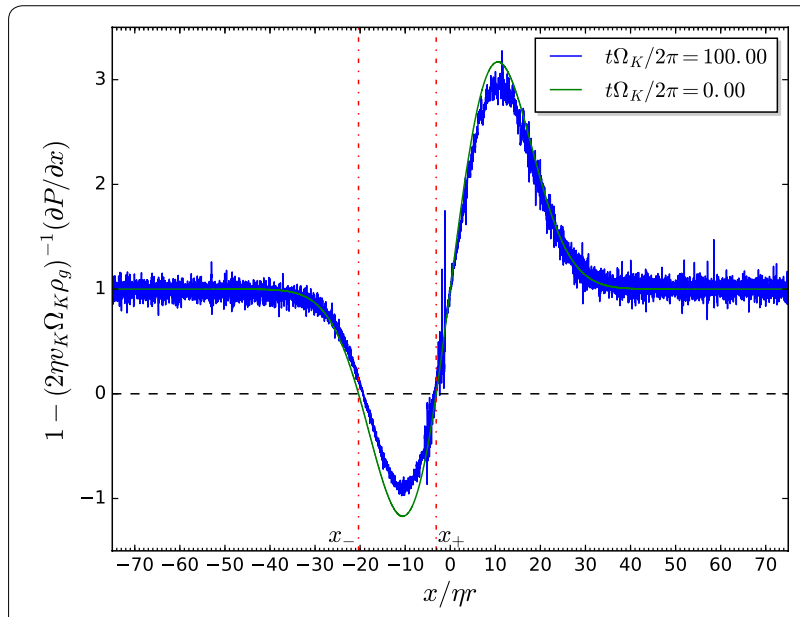

Fig. 1 Radial distribution of the normalized radial pressure gradient force $f$ at the midplane. Initially, there are two points, $x_{-}=-20.36$ and $x_{+}=-3.13$, where $f=0$

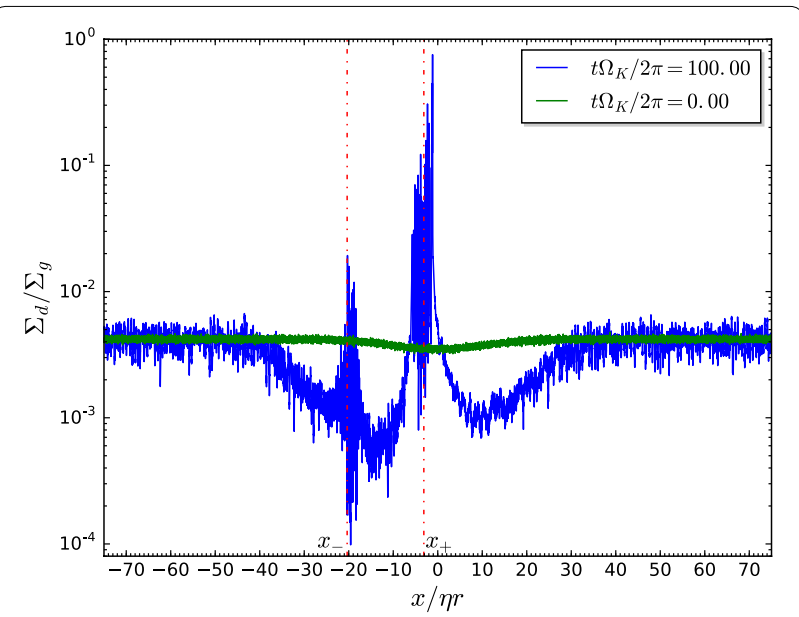

Fig. 2 Radial distribution of metallicity $\Sigma_{d} / \Sigma_{g}$

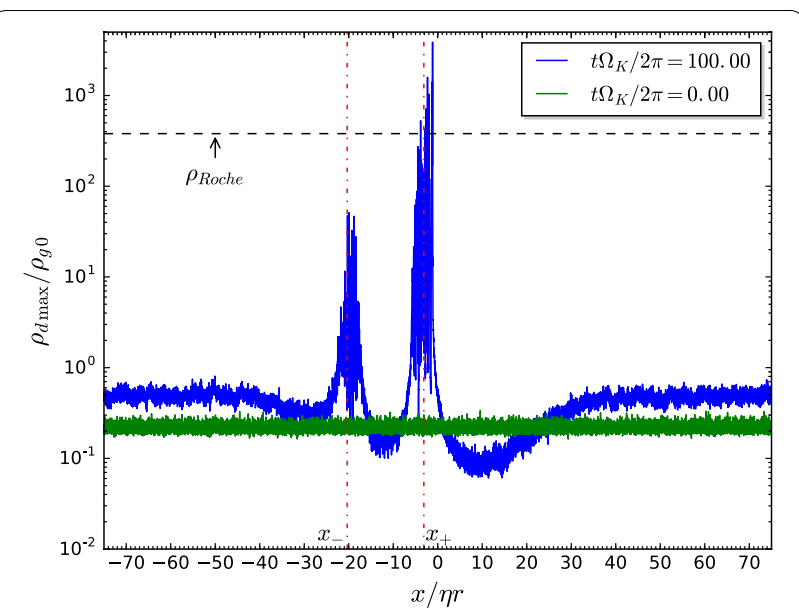

Fig. 3 Radial distribution of maximum dust density $\rho_{d, \text { max. The }}$. horizontal dotted line shows the Roche density $\rho_{\text {Roche }}$ at $1 \mathrm{AU}$ from the star with the solar mass

distribution of $\rho_{d, \max }$, i.e., the maximum dust density among the mesh points of $z$ at each mesh point of $x$. The values of $\rho_{d, \max }$ grow not only around $x_{+}$, where the metallicity grows, but also around $x_{-}$, where the metallicity decreases. Figures 4 and 5 show the dust density distribution around $x_{+}$and $x_{-}$, respectively, in the $x-z$ plane. Note that we assume axisymmetry with respect to the rotation axis; thus, the dust density is independent of $y$.

The dust layer fragments into a number of clumps. The dust densities in the clumps increase not only around $x_{+}$, where the metallicity grows, but also around $x_{-}$, where the metallicity decreases. Although particle-clumping occurs at both null points $x_{+}$and $x_{-}$of the pressure gradient, the maximum density is higher around $x_{+}$because of the high metallicity. As shown in the inlet of Fig. 4, the 


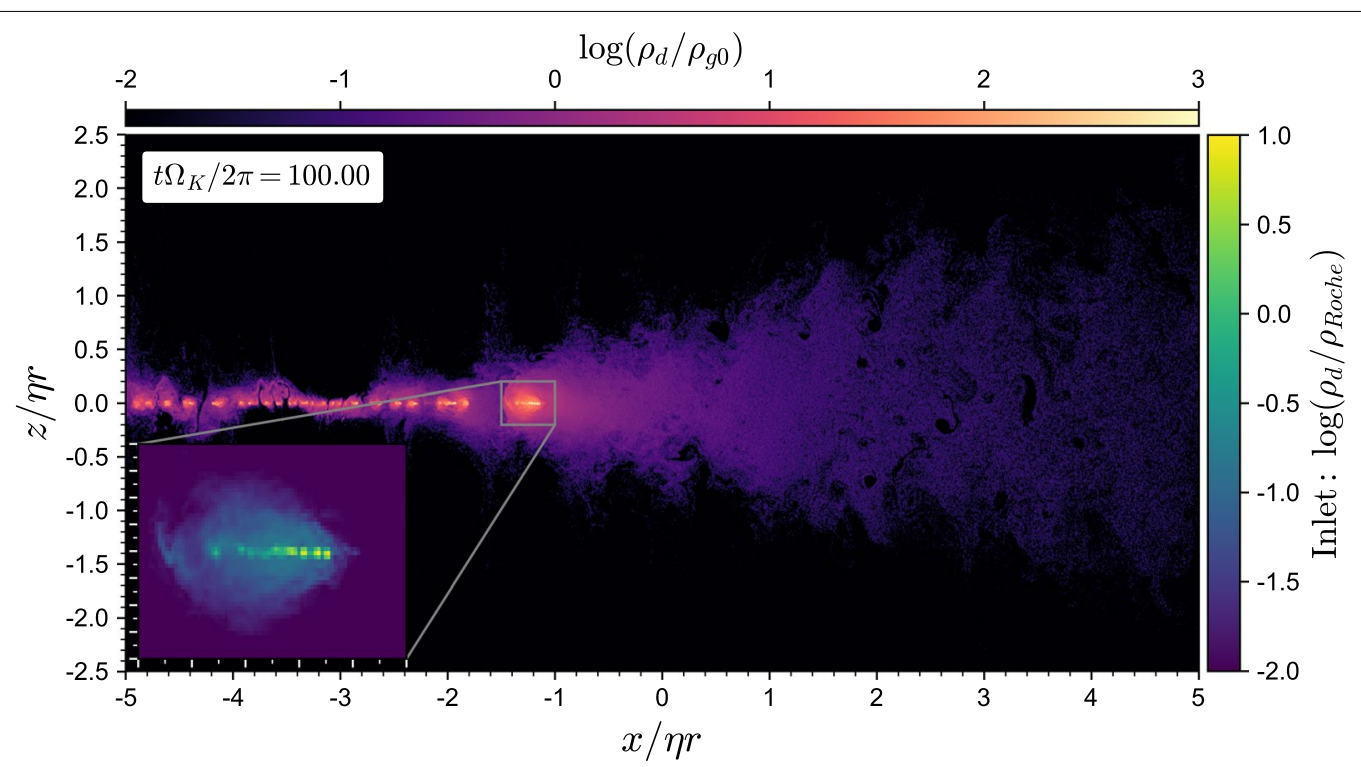

Fig. 4 Dust density distribution around $x_{+}$. The dust density is normalized by $\rho_{g 0}$ (the dust density shown in the inlet is normalized by $\left.\rho_{\text {Roche }}=3.8 \times 10^{2} \rho_{g 0}\right)$

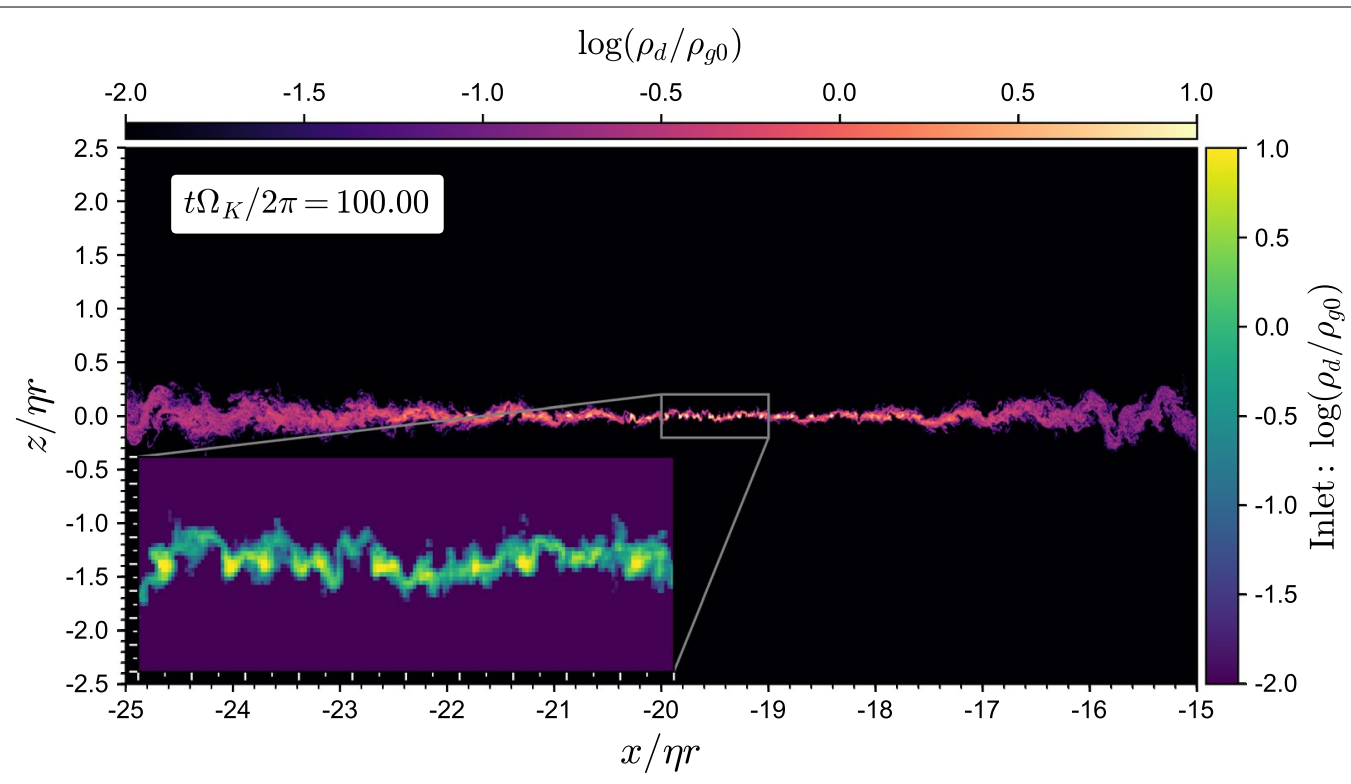

Fig. 5 Dust density distribution around $x_{-}$. The dust density is normalized by $\rho_{g 0}$

maximum dust density exceeds the Roche density after 100 orbits. These high-density clumps are not temporal, but continue to grow in density without migrating radially until the end of our simulation (see Additional files 1, 2 for movies that show the time evolutions; the last frames of these movies correspond to Figs. 4 and 5 , respectively). We can expect further growth of the density for clumps that have a density exceeding the Roche density owing to gravitational instability. Hence, planetesimal formation due to gravitational instability would proceed around $x_{+}$.

Here, we consider the reason why the dust clumping also occurs around $x_{-}$, where the metallicity is low. By comparing Figs. 1, 4, and 5 (and Additional files 1, 2), we can see that the scale height of the dust layer decreases as the absolute value of the normalized pressure gradient force $|f|$ decreases. The scale height is determined by the balance of dust settling toward the midplane due to the 
vertical component of the stellar gravity and the diffusion of dust particles due to turbulence. Thus, the decrease in scale height means that the turbulent diffusion of dust particles is suppressed as the absolute value of the normalized pressure gradient force $|f|$ decreases. Here, we consider the reason for this. According to Weidenschilling (1980), Cuzzi et al. (1993), and Weidenschilling and Cuzzi (1993), the turbulence is caused by the Kelvin-Helmholtz instability due to the difference of the azimuthal velocities between the dust layer around the midplane and outside of the dust layer $\Delta u_{y}$. In this work, the radial pressure gradient is proportional to $f$. Using the formula of Nakagawa et al. (1986) but replacing $\eta v_{K}$ by $f \eta v_{K}$, the absolute value of the gas velocity difference is expressed as $\left|\Delta u_{y}\right| \approx\left[\rho_{d} /\left(\rho_{g}+\rho_{d}\right)\right]|f| \eta v_{K}$ if $\tau_{s}^{2} \ll 1$. Hence, $\left|\Delta u_{y}\right|$ which causes the Kelvin-Helmholtz instability, decreases as $|f|$ decreases. (This is clearly observed in movies that show the time evolution of $\Delta u_{y}$; see Additional files 3,4) The vertical settling of dust particles toward the midplane proceeds where the turbulence is weak. In a region where dust density overcomes the gas density, dust clumps form, and they do not strongly migrate radially because the dust inertia overcomes the gas drag force. Dust particles that are not incorporated in dust clumps are diffused by the gas drag force, and they eventually accrete onto the dust clumps. Our results show that a small radial pressure gradient promotes dust clumping, which is consistent with the results of Bai and Stone (2010c). The development of the dust clumping observed in our simulation may be related to the streaming instability of Youdin and Goodman (2005). We plan to analyze our numerical data in detail in order to elucidate this issue in future work.

We now compare our results with those of Taki et al. (2016). They show in their Fig. 4 that an initially defined pressure bump fades away with time. They explain the cause of the fading as follows. Dust particles migrate outward by acquiring angular momentum from the gas that rotates faster than the particles through the friction force in the region where the radial pressure gradient is positive. Then, the angular momentum of the gas decreases because of the back-reaction. Hence, gas elements in the pressure bump move toward the star, and column density decreases. As a result, the region with positive radial pressure gradient fades away.

However, the back-reaction is effective only the region where $\rho_{d} \gtrsim \rho_{g}$. In our simulation, we take into account the vertical component of the stellar gravity. Dust particles settle around the midplane, and the scale height of the dust layer, where $\rho_{d} \gtrsim \rho_{g}$, is less than a few percent of the scale height of the gas disk. In most of the region of the disk, $\rho_{d} \ll \rho_{g}$, and the back-reaction of migrating dust is not effective for erasing the pressure bump. Thus, the region with $f<0$ is preserved until the dust density exceeds the Roche density around $x_{+}$, as shown in Fig. 1.

In our simulation, the dust clumping proceeds significantly around the null points of the radial pressure gradient $x_{ \pm}$, in contrast to the simulation of Taki et al. (2016). Because Taki et al. (2016) did not include the effect of the vertical gravity, dust density did not increase around the region where the radial pressure gradient is almost zero. In contrast, our simulation, which includes the vertical gravity, shows that the dust density around the midplane grows to form a thin dust layer that fragments into a number of clumps; the dust density of some clumps exceeds the Roche density. Taki et al. (2016) assumed a Stokes number $\tau_{s}$ of 1 instead of 0.01 ; the latter is used in this work. The radius of a solid particle with $\tau_{s}=1$ is a few decimeters; such a large particle may be difficult to form by mutual sticking of silicate dust aggregates (Zsom et al. 2010). However, for completeness, we also performed a simulation for $\tau_{s}=1$ and found that the dust density exceeds the Roche density at $x_{+}$in only five orbits if we take the vertical gravity into account. Additional file 5 shows the results for $\tau_{s}=1$.

Lastly, we note that the assumption of axisymmetry would break because of the Rossby wave instability ( $\mathrm{Li}$ et al. 2000; Meheut et al. 2012a, b; Lin 2012; Ono et al. 2016). A global three-dimensional simulation of gas and dust particles that couple each other by frictional force should be performed in order to elucidate the effect of the pressure bump. Such numerically costly simulations are beyond the scope of this work.

\section{Conclusions}

We investigated the effect of a radial pressure bump on planetesimal formation taking into account stellar vertical gravity. We assumed that there exists a radial pressure bump in a protoplanetary disk. We performed two-dimensional numerical simulations of the motions of dust and gas coupled by the friction force under the assumption of axisymmetry. We used a local shearing box model. We obtained the following results: (1) The column density of dust particles increases around the maximum pressure point. (2) Dust clumps grow around the two points where the radial pressure gradient is equal to zero. (3) The dust density exceeds the Roche density in dust clumps around the maximum pressure point. Planetesimal formation due to gravitational instability will be promoted by a pressure bump. (4) The pressure bump is not erased by the back-reaction of the dust particles concentrating toward the pressure bump, as found in the recent study by Taki et al. (2016), because they did not consider the stellar vertical gravity.

In this study, we neglected the self-gravity of dust particles. If we take the self-gravity into account, the clumps 
with the dust density exceeding the Roche density are believed to collapse further due to self-gravity. Nonaxisymmetric fragmentations in the azimuthal direction will proceed to form a number of clumps that are isolated not only radially but also azimuthally. These clumps will collapse further owing to self-gravity and finally form planetesimals that have a density of the order of the solid density of silicates (but somewhat lower because of the porosity). In order to elucidate the mass distribution of planetesimals, three-dimensional simulations with self-gravity should be performed. Such simulations are beyond the scope of this work and are left for future studies.

\section{Additional files}

Additional file 1. The time evolution of the dust density is shown as a movie, the last panel of which corresponds to Fig. 4 in the main text.

Additional file 2. The time evolution of the dust density is shown as a movie, the last panel of which corresponds to Fig. 5 in the main text.

Additional file 3. The time evolution of $\Delta u_{y}$ is shown as a movie, the region of which corresponds to Fig. 4 in the main text.

Additional file 4. The time evolution of $\Delta u_{y}$ is shown as a movie, the region of which corresponds to Fig. 5 in the main text.

Additional file 5. This file includes results for $\tau_{s}=1$ (Figs. S1-S5).

\section{Authors' contributions}

IO contributed to the computer simulations and creation of figures, and MS drafted the manuscript. Both authors read and approved the final manuscript.

\section{Author details}

${ }^{1}$ Department of Earth and Planetary Sciences, Graduate School of Science, Kyushu University, 744, Motooka, Nishi-ku, Fukuoka 819-0395, Japan. ${ }^{2}$ Department of Earth and Planetary Sciences, Faculty of Science, Kyushu University, 744, Motooka, Nishi-ku, Fukuoka 819-0395, Japan.

\section{Acknowledgements}

This work was supported by JSPS KAKENHI Grant Number 15K05268. Numerical computations were carried out on the Cray XC30 at the Center for Computational Astrophysics, National Astronomical Observatory of Japan.

\section{Competing interests}

The authors declare that they have no competing interests.

\section{Publisher's Note}

Springer Nature remains neutral with regard to jurisdictional claims in published maps and institutional affiliations.

Received: 18 January 2017 Accepted: 6 April 2017

Published online: 18 April 2017

\section{References}

Adachi I, Hayashi C, Nakazawa K (1976) The gas drag effect on the elliptic motion of a solid body in the primordial solar nebula. Prog Theor Phys 56:1756-1771

Bai X-N, Stone JM (2010a) Particle-gas dynamics with Athena: method and convergence. Astrophys J Suppl 190:297-310
Bai X-N, Stone JM (2010b) Dynamics of solids in the midplane of protoplanetary disks: implications for planetesimal formation. Astrophys J 722:1437-1459

Bai X-N, Stone JM (2010c) The effect of the radial pressure gradient in protoplanetary disks on planetesimal formation. Astrophys J 722:L220-L223

Carrera D, Johansen A, Davies MB (2015) How to form planetesimals from mmsized chondrules and chondrule aggregates. Astron Astrophys 579:A43

Chandrasekhar S (1987) Ellipsoidal figures of equilibrium. Dover, New York

Chiang E, Youdin AN (2010) Forming planetesimals in solar and extrasolar nebula. Ann Rev Earth Planet Sci 38:493-522

Cuzzi JN, Dobrovolskis AR, Champney JM (1993) Particle-gas dynamics in the midplane of a protoplanetary nebula. Icarus 106:102-134

Goldreich P, Ward WR (1973) The formation of planetesimals. Astrophys J 183:1051-1061

Haghighipour N, Boss AP (2003) On pressure gradients and rapid migration of solids in a nonuniform solar nebula. Astrophys J 583:996-1003

Hayashi C (1981) Structure of the solar nebula, growth and decay of magnetic fields and effects of magnetic and turbulent viscosities on the nebula. Suppl Prog Theor Phys 70:35-53

Johansen A, Youdin A (2007) Protoplanetary disk turbulence driven by the streaming instability: nonlinear saturation and particle concentration. Astrophys J 662:627-641

Johansen A, Youdin A, Mac Low M-M (2009) Particle clumping and planetesimal formation depend strongly on metallicity. Astrophys J 704:L75-L79

Kokubo E, Ida S (1996) On runaway growth of planetesimals. Icarus 123:180-191

Kokubo E, Ida S (1998) Oligarchic growth of protoplanets. Icarus 131:171-178

Kretke KA, Lin DNC (2007) Grain retention and formation of planetesimals near the snow line in MRI-driven turbulent protoplanetary disks. Astrophys J 664:L55-L58

Li H, Finn JM, Lovelace RVE, Colgate SA (2000) Rossby wave instability of thin accretion disks. II. Detailed linear theory. Astrophys J 533:1023-1034

Lin M-K (2012) Rossby wave instability in locally isothermal and polytropic disks: three-dimensional linear calculations. Astrophys J 754:21

Lyra W, Mac Low M-M (2012) Rossby wave instability at dead zone boundaries in three-dimensional resistive magnetohydrodynamical global models of protoplanetary disks. Astrophys J 756:62

Meheut H, Keppens R, Casse F, Benz W (2012) Formation and long-term evolution of 3D vortices in protoplanetary discs. Astron Astrophys 542:A9

Meheut H, Meliani Z, Varniere P, Benz W (2012) Dust-trapping Rossby vortices in protoplanetary disks. Astron Astrophys 545:A134

Nakagawa Y, Sekiya M, Hayashi C (1986) Settling and growth of dust particles in a laminar phase of a low-mass solar nebula. Icarus 67:375-390

Ono T, Muto T, Takeuchi T, Nomura H (2016) Parametric study of the Rossby wave instability in a two-dimensional barotropic disk. Astrophys J 823:84

Safronov VS (1969) Evolution of the Protoplanetary Cloud and Formation of the Earth and the Planets. NASA Tech. Trans. F-677

Sekiya M (1998) Quasi-equilibrium density distributions of small dust aggregations in the solar nebula. Icarus 133:298-309

Taki T, Fujimoto M, Ida S (2016) Dust and gas density evolution at a radial pressure bump in protoplanetary disks. Astron Astrophys 591:A86

Weidenschilling SJ (1977) Aerodynamics of solid bodies in the solar nebula. Mon Not R Astron Soc 180:57-70

Weidenschilling SJ (1980) Dust to planetesimals: settling and coagulation in the solar nebula. Icarus 44:172-189

Weidenschilling SJ, Cuzzi JN (1993) Formation of planetesimals in the solar nebula. In: Levy EH, Lunine JI (eds) Protostars and planets III. University of Arizona Press, Tucson, pp 1031-1060

Wetherill GW, Stewart GR (1989) Accumulation of a swarm of small planetesimals. Icarus 77:330-357

Youdin AN, Goodman J (2005) Streaming instabilities in protoplanetary disks. Astrophys J 620:459-469

Youdin A, Johansen A (2007) Protoplanetary disk turbulence driven by the streaming instability: Linear evolution and numerical methods. Astrophys J 662:613-626

Zsom A, Ormel CW, Güttler C, Blum J, Dullemond CP (2010) The outcome of protoplanetary dust growth: pebbles, boulders, or planetesimals? II. Introducing the bouncing barrier. Astron Astrophys 513:A57 